\title{
La autonomía de la comprensión humanística
}

\author{
Jorge R. Tagle Marroquín \\ Universidad Autónoma de Zacatecas/ \\ Universidad Autónoma Metropolitana-Iztapalapa \\ jtag@xanum.uam.mx
}

«¿Existen diferencias de fondo más bien que de grado entre la comprensión y saber humanísticos ${ }^{1}$ y la comprensión y saber científico-naturales ${ }^{2}$ ? ¿Tendría que haberlas?». Para muchos profesionales de las humanidades, la respuesta afirmativa a las dos interrogantes anteriores no admite titubeos. "Y así tiene que ser», se nos dirá, pues en un debate de una muy larga historia, que se remonta hasta los inicios de la filosofía en las ideas de Platón y Aristóteles, se ubica el núcleo de las diferencias en la distinción entre explicar un suceso mediante algún mecanismo causal y comprender las motivaciones o finalidad última de lo que ocurre ${ }^{3}$. La dicotomía es clara,

\footnotetext{
1 Utilizo «humanístico» y no más bien «humanista» por una razón: el segundo término tiene usos que lo identifican con el estudio de la cultura antigua y del Renacimiento, o en todo caso limitado al estudio de la cultura literaria, mientras que a mí me interesa un término que se aplique a todo lo que se conoce como las humanidades, incluyendo su relación con las ciencias sociales. «Saber humanístico» se utiliza en lugar de «saber de las humanidades».

2 Podría parecer innecesario añadir «científico» a «natural», un pleonasmo; sin embargo, considero indispensable hacerlo porque al hablar de un saber o conocimiento natural se podría pensar que estoy hablando de saber o conocimiento intuitivo; que no es el caso.

3 Ver von Wright 1971, para una manera de trazar la distinción entre erklaren («explicar») y verstehen («comprender»).
} 
fundamental e inobjetable, se nos dirá, y responde a la diferencia en objetos de estudio entre las humanidades y la ciencia natural. Punto.

A pesar de lo anterior -a pesar de la venerable tradición que ha aceptado, defendido y argumentado que la diferencia entre las humanidades y la ciencia natural es innegable, y aproximadamente corresponde ${ }^{4}$ a la distinción entre comprender versus explicar-, en el presente ensayo me propongo explorar el alcance de la siguiente sugerencia: más allá de la diferencia obvia entre vocabularios respectivos, hay muchas menos diferencias entre el saber científico-natural y el saber humanístico de las que usualmente se aceptan. $\mathrm{Y}$ esto no quiere decir, ni mucho menos es equivalente a afirmar, que hay reducción teórica, metodológica -un saber se reduce a otro, pues a final de cuentas hay un solo método al cual se reduce cualquier otro-, ontológica -un dominio de entidades se reduce a otro dominio de entidades-, o que no tenga sentido hablar de la autonomía conceptual del saber humanístico.

Hay, como defendió Quine en su clásico «Two Dogmas of Empiricism», y puede considerarse como un eslogan que sintetiza la sugerencia que exploro a continuación, continuidad entre la filosofía, el resto de las humanidades, y la investigación científico-natural; $\mathrm{y}$, a mi parecer, esto no es incompatible con la autonomía del saber humanístico. Una interpretación de la distinción entre saber homonómico y saber heteronómico, que argumento largamente más adelante, y que Davidson traza en su «Mental Events», me proporciona una plataforma que me permite explorar una visión com-

\footnotetext{
4 Utilizo «aproximadamente» pues debería ser claro que nuestra capacidad para comprender la conducta y acciones de nuestros semejantes es muchísimo más compleja, completa y extensa que cualquier cosa que se pueda encontrar en las humanidades.
} 
patibilista entre el saber humanístico y el saber científiconatural. Si tomamos aquélla como una distinción útil para representar el saber y el conocimiento humano en su totalidad, independientemente de que se piense que hay diferencias importantes entre los saberes específicos que integran a la totalidad del mismo, entonces podemos pensar a la distinción entre saber humanístico y saber científico-natural como contenida en ella -lógicamente, por supuesto-, y las diferencias no lingüísticas entre saberes son más de grado que de naturaleza, tal y como sucede con aquella otra distinción. La autonomía del saber humanista, consecuentemente, puede replantearse sin tentaciones revisionistas. Paso a los detalles.

\section{El saber humanístico y el debate acerca del naturalismo filosófico}

No está lejos de la verdad afirmar que el debate filosófico contemporáneo respecto de la relación entre el saber humanístico y el científico natural se ha reducido, casi enteramente, incluso por encima de la polémica entre comprender versus explicar, al debate acerca de las limitaciones y fortalezas de lo que se ha llamado «naturalismo filosófico», caracterizado y criticado del siguiente modo por uno de los protagonistas principales de dicho debate:

Necesitamos recuperar la idea aristotélica de que un ser humano normal, maduro, es un animal racional, pero sin perder la idea kantiana de que la racionalidad opera libremente en su propia esfera. La idea kantiana se refleja en el contraste entre la organización del espacio de las razones y la estructura del reino de la ley natural. El naturalismo moderno se olvida de la segunda 
naturaleza; si intentamos conservar el pensamiento kantiano de que la razón es autónoma dentro del esquema de aquella clase de naturalismo desconectamos nuestra racionalidad de nuestro ser animal, que es lo que nos proporciona nuestro asimiento en la naturaleza. El resultado es una tentación de tirar por la borda el pensamiento kantiano y naturalizar nuestra racionalidad al modo como lo hace el naturalismo sin adornos. He descrito eso como decidir no ser parte de esta área de la filosofía (McDowell 1994, 85; la traducción es mía) ${ }^{5}$.

Si entiendo correctamente a McDowell, el naturalismo moderno, y más gravemente para él, el «naturalismo sin adornos» (bald naturalism, en lengua inglesa) ven a la racionalidad humana, el primero, como un tema más de la ciencia, el segundo, no sólo como un tema más de la ciencia sino además, lo más preocupante para él, como un objeto más del mundo físico -reducción ontológica, según la cual no hay sino una sola clase de objetos-. Y si la racionalidad se toma como un objeto más del mundo físico entonces, para McDowell, la racionalidad humana pasa a ser un elemento más de la «estructura del reino de la ley natural», un objeto más explicable y predecible según leyes naturales. Sobre esta característica central del naturalismo sin adornos es muy pertinente traer algunos detalles adicionales a nuestra discusión. Un par de

\footnotetext{
5 El pasaje del cual ofrezco una traducción es el siguiente: «We need to recapture the Aristotelian idea that a normal mature human being is a rational animal, but without losing the Kantian idea that rationality operates freely in its own sphere. The Kantian idea is reflected in the contrast between the organization of the space of reasons and the structure of the realm of the natural law. Modern naturalism is forgetful of second nature; if we try to preserve the Kantian thought that reason is autonomous within the framework of that kind of naturalism, we disconnect our rationality from our animal being, which is what gives us our foothold in nature. The upshot is a temptation to drop the Kantian thought and naturalize our rationality in the manner of bald naturalism. I have described that as opting out of this region of philosophy».
} 
naturalistas contemporáneos nos indican cómo debemos intentar entender la relevancia de la ley natural para la racionalidad humana; el primero es Jaegwon Kim, en la actualidad considerado como una autoridad en metafísica, que en un comentario crítico sobre la filosofía de Davidson caracteriza al naturalismo moderno, conocido como fisicalismo reductivista, y lo contrasta con el naturalismo de Davidson:

El supuesto aceptado previamente por casi cualquier filósofo, y también por psicólogos, que data de muchos años atrás, había sido que aunque los fenómenos mentales ${ }^{6}$ podrían ser distintos de los fenómenos físicos, hay conexiones legaliformes íntimas entre los dos dominios. Teorías pasadas de la relación mentecuerpo -tales como la teoría del doble-aspecto, el paralelismo, el monismo neutral, y la doctrina de la armonía preestablecida- habían sido propuestas sobre la base de una creencia en un sistema omnipresente de correlaciones legaliformes entre la mente y el cuerpo; y la primera teoría de la identidad mente-cuerpo formulada por Herbert Feigl y J.J.C. Smart, la presuponía. La idea misma de «residuos nomológicos», popularizada por estos defensores del fisicalismo reduccionista, implica que hay leyes psicofísicas - «residuales» o de alguna otra forma- que debemos eliminar o en su defecto tomar como leyes básicas irreductibles. La supuesta existencia de estas leyes correlacionales fue lo que hizo posible para nosotros el pensar que el mundo mental y el mundo físico conformasen un todo íntegro y unificado, y que, no obstante poseemos, en tanto personas, tanto un aspecto mental como uno físico, éstos están estrechamente coordinados constriñendo y siendo constreñido por uno y otro (Kim 2003, 118; la traducción es mía) ${ }^{7}$.

Léase «racionales».

7 El pasaje original es el siguiente: «The assumption previously held by almost all philosophers, 
El concepto clave en este pasaje de Kim es «correlaciones legaliformes» (correlation laws es la expresión para ello en lengua inglesa) que busca extenderse sobre cualesquiera «conexiones íntimas» entre lo físico y lo mental. La aspiración de este naturalista sin adornos, también conocido como «fisicalista reductivista», sería encontrar las correlaciones legaliformes que coordinan a cada integrante del dominio de lo mental, en el cual opera la racionalidad, con los elementos del dominio de la ley natural. Lo mental-racional y lo físico, si bien coordinados, constituirían «un todo unificado e integrado» y nada quedaría fuera de la ley natural, como lo teme McDowell. Alternativamente, si pasamos a segundo plano las correlaciones legaliformes de las que habla Kim, podría pensarse la coordinación entre lo físico y lo racional en otros términos, tal y como lo adelanta Brian Loar, otro filósofo fisicalista:

La cuestión importante es si es el caso que, independientemente de si nos referimos a proposiciones, las condiciones que delimitan a la racionalidad se corresponden estructuralmente con las condiciones puramente físicas que delimitan a los estados físicos. Y no hay ningún impedimento para ello. Para que los es-

\footnotetext{
and also psychologists, going back many years, had been that although mental phenomena might be distinct from physical phenomena, there are intimate lawful connections between the two domains. Hoary mind-body theories -such as the double-aspect theory, parallelism, neutral monism, and the doctrine of preestablished harmony- had been erected on the basis of a belief in a pervasive system of lawful correlations between mind and body; and the early mind-body identity theory formulated by Herbert Feigl and J.J.C. Smart, had presupposed it. The very idea of "nomological danglers", made popular by these advocates of reductionist physicalism, implies that there are psychophysical laws -"dangling" or otherwise- that we must either eliminate or else take as irreducible basic laws. The supposed existence of these correlation laws was what made it possible for us to think that the mental world and the physical world make up a unified and integrated whole, and that although we as persons have both a mental and a physical aspect, they are closely coordinated with each constraining and being constrained by the other».
} 
tados-tipo físicos $x$ y y estén relacionados tal y como la teoría dice que las creencias de que $p$ y $q$ y la creencia de que $\sim p$ están contrafácticamente relacionadas será el caso, al menos parcialmente, que si $x$ fuese a suceder entonces $y$ no sucedería. Las condiciones que delimitan la racionalidad generan una amplia red de relaciones contrafácticas tales entre estados físicos, con el efecto, después de todo, de describir un sistema de estados-tipo físicos cuyas interrelaciones contrafácticas reflejan las relaciones lógicas relevantes entre creencias y deseos (Loar 1981, 23; la traducción es mía $)^{9}$.

Loar con optimismo pasa a hablar ya no de correlaciones legaliformes entre lo físico y lo mental, sino de "correspondencias estructurales» entre las condiciones que delimitan a la racionalidad («the constraints on rationality», en lengua inglesa) y las condiciones puramente físicas que delimitan a lo físico; las correlaciones legaliformes no desaparecen sino que pasan a segundo plano y se analizan contrafácticamente. El reino de la ley natural ganaría en profundidad según la perspectiva funcionalista que impulsan Kim, Loar, Lewis, entre otros, al analizar las condiciones que delimitan a la racionalidad, lo que posteriormente McDowell llamará «el espacio de las razones», en términos contrafácticos. Lo que Loar asevera es que quienquiera que afirmase una conjunción no

\footnotetext{
$8 \quad$ El pasaje original de Loar es el siguiente: «The important question is whether, regardless of whether propositions are referred to, the constraints on rationality correspond structurally to purely physical constraints on physical states. And there is no impediment to that. For physical state-types $x$ and $y$ to be related as the theory says the belief that $p$ and $q$ and the belief that $\sim p$ are counterfactually related is, in part, for it to be the case that if $x$ were to occur then y would not occur. The rationality constraints generate a vast network of such counterfactual relations among physical states, ultimately with the effect of describing a system of physical states types whose counterfactual interrelations mirror the relevant logical relations among beliefs and desires».

9 En este punto no se ha avanzado mucho; lo mismo ha defendido D. Lewis en, por ejemplo su 1994, y muchos lo siguen a pie juntillas.
} 
podría afirmar simultáneamente la negación de uno de los conyuntos, y esto estaría reflejando una condición o condiciones puramente físicas que delimitan los estados físicos que subyacen a los estados mentales -si se tuviera un estado físico (el relacionado con la conjunción) no se podría tener el otro (el relacionado con la negación del enunciado simple) y viceversa. Se generaría una vasta red de información contrafáctica sobre estados racionales que se piensa que tendría la correspondiente vasta red de información contrafáctica sobre estados físicos -reflejo descrito en términos puramente físicos que haría posible una explicación en términos de condiciones físicas contrafácticas de la racionalidad y vida mental humanas ${ }^{10}$.

Si así fuese, si estuviese a la mano la reconstrucción física de lo que constituye a la racionalidad, y si el «espacio de las razones» de McDowell se pudiese analizar contrafáctica y legalmente, entonces la autonomía del saber humanístico, y buena parte de las ciencias sociales, tan sólo sería un wishful thinking de los profesionales de las humanidades.

Sea que se analicen contrafácticamente o no, las correlaciones legaliformes, tanto naturalistas sin adornos como sus oponentes, operan bajo el supuesto de que algo hay separado, incompatible, y la tarea por delante es mostrar cómo juntar eso que está dividido o, en su defecto, disolver el mal-

\footnotetext{
10 Estas condiciones -o información- contrafácticas sobre estados físicos bien pueden entenderse como condiciones de aplicación de conceptos y vocabulario mental en términos de condiciones de aplicación de vocabulario físico. El ejemplo de Loar nos indica el camino a seguir: una creencia en que $p$ y $q$ se analizaría en términos de sus condiciones fácticas y contrafácticas de aplicación -no de aceptación o justificación específica-. A diferencia de, por decir algo, lodo, en donde tan sólo se requiere que se satisfagan las condiciones de ser agua y tierra mezcladas, para el caso de $p$ y $q$ también se tendrían que contemplar aplicaciones contrafácticas en las cuales la creencia $q$ no es sino la creencia $p$ puesta en un idioma distinto $\mathrm{y}$, por ende, los estados mentales, $\mathrm{y}$ las bases físicas respectivas, no son dos sino una. Todas estas peculiaridades serían parte de las condiciones de aplicación del vocabulario físico.
} 
entendido de que hay dos dominios irreconciliables ampliamente separados. No comparto este supuesto, no creo que se pueda juntar lo que previamente fue separado. Pero primero veamos cómo McDowell intenta reconciliar a los oponentes.

Lo que McDowell le opone a una y otra clase de naturalismo es una concepción de la racionalidad humana según la cual más que un objeto o una sustancia con cierta manera de operar -conducirse, comportarse- encontramos una manera de actuar entendida como ubicarse en un espacio de razones («space of reasons» en lengua inglesa), «un espacio lógico que está organizado a partir de relaciones de justificación entre sus integrantes»(McDowell, 2004; 92). Estas relaciones de justificación entre los integrantes del reino de lo mental o espacio de las razones representan para McDowell, a diferencia de Loar, una razón para no ser optimistas respecto del poder explicativo de las correlaciones legaliformes: «La estructura del espacio de las razones tercamente se resiste a ser incorporada dentro de un naturalismo que concibe a la naturaleza como el reino de la ley» (McDowell 1994, 73; la traducción es mía $)^{11}$. Las correlaciones legaliformes no son suficientes para explicar las relaciones de justificación, según McDowell. Si fuera el caso, diría una réplica al naturalismo sin adornos en el espíritu de lo que defiende McDowell, la correcta descripción de la estructura del reino de la ley natural parecería requerir de una coordinación tan precisa con la descripción de las condiciones que delimitan a la racionalidad que, so pena de convertirse en información necesaria pero insuficiente, es difícil pensar cómo llevar a cabo tal cosa sin presuponer lo que se quiere caracterizar, ya sea correlaciones legaliformes

\footnotetext{
${ }^{11}$ El pasaje original es el siguiente: «The structure of the space of reasons stubbornly resists being appropriated within a naturalism that conceives nature as the realm of law».
} 
que contienen vocabulario propio del espacio de las razones sin elucidación física ulterior, o condiciones que delimitan la racionalidad en vocabulario puramente de razones. Con optimismo se presupone que las condiciones de aplicación de los conceptos y vocabulario mentales pueden ser descritas, sin residuo alguno, completa y exhaustivamente, en vocabulario y conceptos físicos o físicamente elucidables.

Esto, o algo muy cercano a ello, es o sería autonomía para muchos que simpatizan con el kantismo de McDowell. Parte, sin embargo, de la suposición de que lo que está separado, está muy bien así, y lo más que se puede hacer es tratar de reconciliar el «espacio de las razones»y «la estructura del reino de la ley natural»12. Si bien, al igual que McDowell, no veo cómo un espacio lógico que hace posible relaciones de justificación pueda reducirse a, identificarse con o eliminarse absolutamente a favor de «condiciones que delimitan lo físico descritas en vocabulario puramente físico», no estoy convencido de que ésta sea una buena manera de caracterizar no sólo el tema de la racionalidad versus la ley natural, sino además el disciplinario con el cual se encuentra estrechamente relacionado. Sólo si hay separación se requiere de reconciliación, pero no es claro que haya separación en el punto de partida, o al menos eso argumentaré más adelante.

En todo caso, se puede pensar que tanto el naturalismo sin adornos como el naturalismo liberal que propone McDowell comparten la suposición de que algo está segmentado en al menos dos grandes partes. De lo que se trata es,

\footnotetext{
12 Véase, si no, lo siguiente: «What I am suggesting could be put in terms of a task for philosophy, to effect a reconciliation [...] My proposal is that we should try to reconcile reason and nature [...]» (McDowell, 1994; 85-86). («Lo que estoy sugiriendo podría ponerse en términos de una tarea para la filosofía, llevar a cabo una reconciliación [...] Mi propuesta es que deberíamos intentar reconciliar razón y naturaleza [...]»).
} 
para el primero, de acercar al no naturalista a una posición en donde pueda percatarse de que el reino de la ley natural se sobrepone, y puede elucidar adicionalmente, a la racionalidad. O bien, mostrar que si bien, como defiende McDowell, el dominio de las razones, para utilizar su terminología, opera independientemente en su propio espacio, se sobrepone al reino de la ley natural (está colocado por encima de él). En el primer caso, esa sobreposición hará posible una reducción -las condiciones de aplicación del vocabulario y conceptos físicos se corresponden con las condiciones de aplicación del vocabulario y conceptos no físicos- mientras que en el segundo se permitirá darle un asimiento, en el reino de la ley natural, a la esfera de la racionalidad. Este reino de la ley natural, en suma, se puede concebir a la manera de una estructura que mantiene en pie a la esfera de la racionalidad, o como el esqueleto que le da fuerza y sustento a lo mental.

\section{La distinción entre saber homonómico y saber heteronómico}

El siguiente pasaje de Davidson es iluminador respecto de un contenido que se le puede atribuir a una distinción tal, las razones para aceptarla, así como los conceptos centrales de una concepción compatibilista del saber científico-natural y humanístico:

En nuestro trato diario con sucesos y acciones que deben preverse o entenderse, forzosamente hacemos uso de la generalización esquemática y sumaria, pues no conocemos una ley más precisa; o bien, de conocerla, carecemos de una descripción de los sucesos particulares en los que estamos interesados que mostrara la per- 
tinencia de la ley. Pero hay una distinción importante que debe hacerse dentro de la categoría de la regla práctica burda. Por una parte, hay generalizaciones cuyas instancias positivas nos dan pie para creer que la generalización misma podría mejorarse añadiéndole otras estipulaciones y condiciones formuladas en el mismo vocabulario general que la generalización original. Tal generalización apunta a la forma y al vocabulario de la ley acabada: podemos decir que es una generalización homonómica. Por otra parte, hay generalizaciones que, instanciadas, pueden darnos razón para creer que se está trabajando con una ley precisa, pero que sólo puede enunciarse si cambiamos a un vocabulario diferente. Podemos llamar heteronómicas a tales generalizaciones.

Supongo que la mayor parte de nuestro saber práctico (y científico) es heteronómico. Ello se debe a que puede esperarse que una ley sea precisa, explícita y, hasta donde sea posible, sin excepciones, sólo si extrae sus conceptos de una teoría comprehensiva cerrada (Davidson, 1970b; 277-278. Traducción inglésespañol de Olbeth Hansberg, José Antonio Robles y Margarita Valdés).

A continuación propongo y defiendo una interpretación de este pasaje de Davidson, e identifico seis aspectos en la distinción homonómico/heteronómico, aspectos que según mi argumentación nos permiten esbozar una descripción del saber en su totalidad, y a su interior del saber científico-natural en continuidad con el saber humanístico y sus relaciones de justificación.

i) En el pasaje citado, Davidson habla de sucesos y de acciones que debemos explicar o prever, o al menos que nosotros consideramos como nuestro deber explicar y prever. $\mathrm{Y}$ tanto la postulación de sucesos como la de acciones me parece enteramente apropiada en este contexto, pues se re- 
conoce que lo no intencional, los sucesos según una concepción ampliamente aceptada aunque aun bajo discusión ${ }^{13}$-la erupción de un volcán, la caída de un satélite, la circulación de la sangre en el cuerpo, son ejemplos-, tanto como la bien conocida acción intencional que difícilmente tenemos que ilustrar con ejemplos -ejemplos todos objeto de estudio del saber humanístico en el derecho, la literatura, la antropología, la historia, la lingüística y la filosofía-, son los objetos bajo estudio en nuestro saber práctico y científico.

A partir de esta distinción parecería enteramente apropiado plantear la distinción entre comprender y explicar, sobre la base de que los sucesos se explican y prevén mientras que las acciones solamente se comprenden. Pero no es claro que esto tenga que ser así necesariamente, pues también hay acciones que se prevén ${ }^{14}$.

ii) Davidson habla de «sucesos y acciones que deben preverse o entenderse», y este deber también apunta a un aspecto importante, común tanto al saber científico-natural como al saber humanístico: ambos responden a lo que podríamos denominar «exigencias de inteligibilidad», a una necesidad práctica o intelectual de, llegado el caso, hacerse inteligible lo que sucede, sea esto entendido como sucesos o como acciones intencionales. Más adelante interpretaré este deber más bien como un interés con ciertas características; pues si bien hay

\footnotetext{
13 Ver Davidson 1970a y 1970b; para un discusión reciente sobre la ontología de sucesos, puede verse Simons 2003.

14 Nuestra práctica cotidiana habla de prever acciones también: «vi venir tu respuesta», «sabía que eso pasaría», y muchos otros ejemplos similares. El entendimiento de un mundo concebido como totalidad de sucesos, si fuera cierta la dicotomía entre explicar-prever y comprender, supondría que el entendimiento de sucesos opera independientemente de otras clases de entendimiento, como el entendimiento lingüístico y no lingüístico; como si existiesen clases de entendimiento al modo de módulos o bloques independientes que la mente humana pone en juego dependiendo del objeto en cuestión. Dudoso, por decir lo menos.
} 
teorías de los deberes epistémicos, encuentro menos problemático hablar de intereses epistémicos. Pero esto aparecerá más adelante. Reconozco que este deber en común todavía puede representar muy poco para muchos críticos del naturalismo, si de propiedades compartidas entre saber científiconatural y saber humanístico se trata, pues se pensará que las diferencias más allá del vocabulario aparecerán justo en el momento en que se pongan a discusión las explicaciones que se buscan en cada caso, en un caso explicación causal mientras que en el otro explicación que apela a motivaciones. No obstante se crea en diferencias más allá del vocabulario entre tipos de explicación entre saber humanístico y saber científico-natural, a pesar de la presencia de algunas propiedades compartidas, este deber en común me parece inobjetable. Es en los detalles de las maneras en las cuales cumplimos con ese deber donde parecen surgir las diferencias.

iii) Lo que he llamado «exigencias de inteligibilidad» posee dos aspectos, por un lado, entender el por qué ocurre un suceso o una acción y, por el otro, anticiparse y/o pronosticar la aparición de una acción o un suceso ${ }^{15}$. Se busca inteligibilidad respecto del pasado y el presente así como del futuro. Y respecto de esto es importante distinguir la exigencia de inteligibilidad del presente de la del pasado, pues a diferencia de las ciencias sociales, como por ejemplo la ciencia política, la economía, la psicología o incluso la sociología -sobre todo las que usan y aprovechan métodos estadísticos-, que sí se ocupan del presente e incluso tienen ambiciones prospectivas, en las humanidades el objeto de estudio está constituido predo-

15 Por supuesto que no cabría hablar del mismo suceso o la misma acción, si nos atenemos a un criterio de individuación de acciones y sucesos que contiene un aspecto temporal. 
minantemente por acciones y pensamiento pasado -si bien éste no es una acción intencional.

En el caso del saber científico-natural, por otra parte, la exigencia de inteligibilidad parecería ser indiferente respecto de consideraciones de pasado, presente y futuro. Pero esto está lejos de ser el caso, pues hay fenómenos estudiados por la física que son claramente temporales: el big bang, la formación y desaparición de cuerpos celestes, la expansión y dinámica del universo, la evolución y muchos más. Llamemos a esto «la temporalidad del objeto de estudio y la exigencia de inteligibilidad». La creencia de que tanto el objeto de estudio como la exigencia de inteligibilidad son atemporales me parece en todo caso una idealización metodológica que responde a nuestros intereses; preferimos pensar que el objeto de estudio -sea acción, sea suceso- se comporte de manera uniforme a lo largo del tiempo, o que las acciones o sucesos similares se comporten y comportarán de manera similar. Del mismo modo, preferimos pensar que nuestra exigencia de inteligibilidad siempre aparece del mismo modo, que entendemos y esperamos entender del mismo modo, independientemente de nuestros intereses.

iv) Un cuarto aspecto identificado por Davidson en su «Mental Events» es el contraste entre generalización heteronómica y ley acabada; una generalización puede ser desde una «regla práctica burda», como él las llama, como por ejemplo «comer demasiado provoca indigestión», pasando por generalizaciones formuladas en vocabulario que contiene términos no cuantitativos, o en todo caso no técnicos, cuyas características frontales tienen que ver con ser esquemáticos y sumarios, sin excluir a vocabulario propio de la motivación humana, por ejemplo «toda persona mayor de edad es legalmente responsable de sus actos», hasta gene- 
ralizaciones que parecen «apuntar» a leyes precisas como «altos niveles de circulante provocan picos inflacionarios»o «niveles por encima de lo normal de pentotal sódico (tiopentato de sodio) inhiben la experiencia de dolor»; todas ellas, si interpreto correctamente el pasaje de Davidson, parecen excelentes candidatas a generalizaciones heteronómicas.

Generalizaciones, por su parte, cuyo rasgo más notable radica en estar expresadas en vocabularios explícitos y precisos en un sentido cuantitativo, pertenecientes a teorías acabadas y completas -experimentalmente probadas, empíricamente confirmadas, conceptualmente cuidadas (sin conceptos primitivos), como, por ejemplo, las que integran la teoría mecánica estadística de los gases-, pueden denominarse «generalizaciones homonómicas o leyes acabadas». Llamemos a este aspecto de la distinción «entendimiento mediante uso de generalizaciones».

Creo que aquí podemos plantear una distinción o varias distinciones que nos ofrecen una idea más precisa de lo que he llamado entendimiento mediante uso de generalizaciones. Por un lado, tendríamos una visión doblemente restrictiva, la cual efectivamente excluye de las humanidades tanto a las generalizaciones heteronómicas como a las homonómicas. Por otro lado, tendríamos una visión restrictiva simple cuando tan sólo se excluye a las generalizaciones homonómicas y a las generalizaciones heteronómicas cuantitativas de las humanidades, y de lo que McDowell llama «el espacio lógico de relaciones de justificación». Esta visión estaría respaldada por algo que se antoja como un hecho innegable, las generalizaciones homonómicas son los candidatos idóneos para conformar «la estructura del reino de la ley natural». En una visión tolerante, por último, tendríamos a cualquier generalización heteronómica como elemento integral de las huma- 
nidades, como la estrategia central, junto con las relaciones de justificación, que nos permiten comprender acciones y sucesos. Nótese, no obstante, que las generalizaciones heteronómicas bajo clarificación conceptual, experimentalmente probadas y comprobadas en una teoría física bajo desarrollo, que bien podrían llamarse una generalización homonómica en ciernes, no parecen estar excluidas en la visión tolerante.

¿Debe pensarse que en el saber humanístico en general -y en el espacio de las razones de McDowell- estarían fuera de lugar las generalizaciones heteronómicas, y en general el entendimiento mediante empleo de generalizaciones? ¿Sería aceptable tal cosa? ¿No habría lugar para la inducción y la comprensión mediante subsunción, «instanciación» o ejemplificación en las humanidades, modos específicos de desplegar generalizaciones -así sólo sea de manera implícita-? Está lejos de ser claro que la ejemplificación o la inducción no aparezcan repetidamente en el saber humanístico. Uno pensaría que incluso el razonamiento analógico, tan utilizado en las humanidades, en tanto razonamiento que busca la comprensión mediante la identificación de similitudes entre objetos dispares y la aplicación de una estrategia o hipótesis explicativa común, quedaría excluido si la generalización heteronómica quedara fuera del entendimiento propio del saber humanístico; peor les iría a la inducción enumerativa y a la ejemplificación.

Es difícil, sin embargo, aceptar algo así, pues es como si la comprensión del personaje literario, o en muchos casos de personajes históricos, no se llevara a cabo a partir de la construcción pormenorizada de un agente descrito de cierta manera (general), sensible a motivaciones, razones, valores y 
exigencias concretas ${ }^{16}$, lo cual nos echa luz sobre el comportamiento del personaje literario o histórico concreto, para no hablar de la explicación causal de la acción -que efectivamente acepta $\mathrm{McDowell}^{17}$-. El análisis literario de un personaje a partir de la recolección de rasgos de casos específicos y su interacción con otros seres humanos, hasta construir un modelo que se aplica a partir de la satisfacción de un número de condiciones: el Dr. Peter Kien y el sabio moderno insensible y poco o nada comprometido socialmente en la novela de Canetti; y otras estrategias explicativas, como la genealogía o la reconstrucción histórica, también comparten aspectos del razonamiento analógico, y por ende, de la generalización heteronómica.

En su contribución al volumen sobre la filosofía de Donald Davidson, de la biblioteca de los filósofos vivos de Schilpp y Hahn, B. Ramberg comenta el papel de la generalización heteronómica en la filosofía de la mente de Davidson, y sugiere algo que es útil para profundizar en nuestra comprensión de lo que está en juego en la distinción heteronómico/homonómico:

¿Quizá, no obstante, la heteronomía de las generalizaciones en las ciencias naturales es una especie de defecto, una expresión de nuestro fracaso en alcanzar, todavía, la clase de unidad a la cual

\footnotetext{
16 Claro que aquí habría consideraciones acerca de actuar racionalmente y responder a ideales de la misma clase, y eso está muy bien, pues no defiendo la idea de que lo que Davidson llama «el ideal constitutivo de la racionalidad» (ver 1970b; 223) se reduzca o identifique con un número de generalizaciones heteronómicas. O peor aún, que eso distinga al saber humanístico del saber científico-natural; claro que no. Mi interés en este ensayo es señalar la continuidad, no enfatizar las diferencias que son bien conocidas.

17 Ver 2004; 92: «We need not see the idea of causal linkages as the exclusive property of naturalscientific thinking». («No necesitamos tomar a la idea de eslabones causales como propiedad exclusiva del pensamiento científico-natural»).
} 
la investigación científico-natural aspira? (Ramberg 1999, 604; la traducción es mía). ${ }^{18}$

El naturalista sin adornos, o incluso el moderno, podría pensar que lo que Ramberg pregunta retóricamente en realidad es el caso para ellos, pues hay que sobreponerse al nivel de la mera generalización heteronómica y pensar en los detalles que estarían ausentes, pero que de conseguirse permitirían disponer de la generalización homonómica -vocabulario y condiciones descritas en términos de la teoría completa-. ¿Será cierto esto? No creo que lo podamos poner en duda respecto de teorías físicas inacabadas que aún contienen vocabulario ajeno a la teoría completa, es decir, teorías de la física que todavía descansan en generalizaciones heteronómicas. Sin embargo, respecto de la mayoría de las generalizaciones heteronómicas del saber humanístico, social y buena parte del natural, como lo dice Davidson y ha sido ampliamente comentado, no veo cómo eso podría ser el caso, so pena de cambiar de tema. $\mathrm{Y}$ en todos esos casos no nos interesa cambiar de tema, estamos interesados en un tema y por eso utilizamos la generalización y el vocabulario que usamos, incluso en la investigación científico-natural no física ${ }^{19}$. Bajo el número de referencia 10/H0708/16, por ejemplo, encontramos un proyecto de investigación del instituto de psiquiatría

18 El pasaje original de Ramberg es el siguiente: «Perhaps, though, the heteronomy of generalizations in natural science is a kind of deficiency, an expression of our failure to have, as yet, achieved the sort of unity to which natural-scientific enquiry aspires?».

19 Hay quienes piensan, por ejemplo G.L. Herstein, que efectivamente cualquier generalización heteronómica «nunca puede levantarse por encima del nivel común y corriente aunque suficiente» («rough and ready»; 2005, 53) de la regla práctica burda, lo cual me parece una mala interpretación del concepto de generalización heteronómica de Davidson a la luz de lo que Davidson mismo dice en su texto. 


\section{del King's College, London y el NHS, cuya descripción es del modo siguiente:}

Evaluaremos los procesos cerebrales que se piensa subyacen y manejan a la anorexia nerviosa en una sola sesión de estimulación magnética transcraneal repetida (EMTr). Este procedimiento es ampliamente utilizado en la investigación e involucra estimular partes del cerebro con ondas magnéticas emitidas al colocar el cráneo dentro de una jaula. También se les solicitará a los participantes completar una entrevista de investigación enfocada en sus hábitos alimenticios, un número de cuestionarios de investigación (enfocados en sus estados de ánimo, estrés y síntomas de ansiedad) y tareas neuropsicológicas (acertijos cerebrales) antes y después de la sesión de EMTr. Los participantes serán confrontados con estímulos alimenticios (alimentos reales y un video) y llevarán a cabo una serie de estimaciones respecto de ellos ${ }^{20}$.

Difícilmente podríamos encontrar un mejor caso en donde opere de manera conjunta, por un lado, el saber heteronómico acerca de estados de ánimo, estrés y ansiedad, actitudes hacia distintos alimentos, y desórdenes de la alimentación que varían con actividad cerebral, y, por el otro, el saber (potencialmente) homonómico acerca de fuerzas magnéticas y su interacción con la estructura neurofisiológica del cerebro.

\footnotetext{
$20 \quad$ El pasaje original es el siguiente: «We will assess the brain processes thought to underlie and drive anorexia nervosa with a single session of repetitive Transcranial Magnetic Simulation (rTMS). This procedure is widely used in research and involves stimulating parts of the brain with magnetic waves delivered by holding a coil to the skull. Participants will also be asked to complete research interview focusing on their eating habits, a number of research questionnaires (focusing on mood, stress and anxiety symptoms) and neuropsychological tasks (brain puzzles) before and after the rTMS. Participants will be presented with some food stimuli (real foods and a film clip) and will complete a number of ratings in relation to these».
} 
No tendría interés alguno para nosotros, interesados (o preocupados) en los desórdenes psicológicos y sus repercusiones en la vida cotidiana, si todo se limitara a las interacciones de ondas magnéticas y sustancias químicas operando en el cerebro humano o, alternativamente, a la mera presencia de distintas clases de alimentos y las reacciones de una $\mathrm{u}$ otra persona -en ambos casos, generalizaciones heteronómicas operando-. ¿Se encontraría esto definitivamente fuera del espacio de las razones de McDowell?

Llamemos a este aspecto de la distinción homonómico/ heteronómico «la ubicuidad de la generalización heteronómica».

v) La aplicación de una generalización, no importa si homonómica o heteronómica, a un caso específico, como Davidson lo indica en el pasaje de su «Mental Events» citado, dependerá de estipulaciones e idealizaciones respecto del objeto de estudio, las cuales incluyen, tanto para el caso de la generalización homonómica como para la generalización heteronómica, descripciones apropiadas de las condiciones específicas en las cuales se localiza el suceso bajo explicación en términos de vocabulario perteneciente a la teoría misma. Para el caso de la generalización heteronómica sucederá, mutatis mutandis, algo similar, aunque en este caso la descripción de condiciones de aplicación de la generalización descansará en descripciones formuladas en vocabulario no técnico e incluso común y corriente. Denominaré a esto la «ubicuidad de las condiciones de aplicación».

vi) En tanto la distinción entre generalización homonómica y generalización heteronómica no indica una diferencia nítida y excluyente entre tipos de saber -científico y práctico, como lo indica el pasaje de Davidson-, entonces podemos sugerir la idea de que el vocabulario propio de las humani- 
dades, aquel que tiene que ver con motivaciones, propósitos, razones y racionalidad tan sólo posee diferencias de grado y profundidad con los vocabularios físico-funcionales, aquellos que tienen que ver con teorías físicas. En el pasaje de Davidson citado destaca un aspecto de la generalización heteronómica y las explicaciones producto de su aplicación, a saber, que a menos que no nos interese formular dicha generalización en un vocabulario diferente por un número de razones, en algunos casos se encuentran en transición hacia el vocabulario, conceptos, saber y explicaciones homonómicas o, en todo caso, para un número muy reducido de teorías físicas, ya ahí -como la teoría mecánica estadística de los gases.

Dice Davidson que podemos pensar que hay una ley precisa operando detrás de una generalización heteronómica, pero que tal cosa podría sacarse a la luz solamente si se cambiara a un vocabulario diferente -y nos interesase hacer tal cosa, yo agregaría, pero por lo regular no nos interesa-. Deseamos permanecer en un nivel explicativo en el cual ciertos conceptos y cierto vocabulario son vitales para nuestros intereses, y no deseamos movernos a otro nivel. Recuérdese el pasaje:

Por otra parte, hay generalizaciones que, instanciadas, pueden darnos razón para creer que se está trabajando con una ley precisa, pero que sólo puede enunciarse si cambiamos a un vocabulario diferente. Podemos llamar heteronómicas a tales generalizaciones.

A la insistencia en permanecer en cierto nivel de explicación, a explicar mediante la aplicación de generalizaciones heteronómicas ignorando formulaciones legales precisas, 
cuando las hubiese, la denominaré «la subordinación de la generalización al interés». Pueden o no pueden existir las leyes precisas, las aplicaciones exitosas de las mismas, y lo que Davidson llama instancias pueden llevarnos a sospechar la existencia de dichas leyes, pero en la gran mayoría de los casos de saber práctico y científico no nos interesa intentar explicar el objeto bajo estudio mediante esa ley precisa, independientemente de si se tienen a la mano descripciones del objeto y las condiciones de aplicación de la ley precisa que sean compatibles con ésta ${ }^{21}$.

\section{Intereses epistémicos}

Pasemos ahora al tema de los intereses que parecen subyacer a la búsqueda de generalizaciones, saber o conocimiento. Necesitamos algunas razones, independientemente de lo persuasivo -la que sea- de la distinción homonómico/ heteronómico y los intereses ahí identificados, para aceptar la importancia, relevancia y rol de éstos, sean epistémicos o prácticos.

Se han propuesto varios argumentos para defender la presencia de intereses prácticos en el conocimiento. Uno de éstos (ver Stanley, 2005) consiste en una serie de experimentos mentales interconectados que giran alrededor de la aseveración siguiente: cuando hay mucho en juego en un sentido práctico (high stakes es la expresión lingüística en inglés), principalmente en un sentido económico, la atribución de saber y conocimiento -por ejemplo, «Ana sabe que la sucur-

\footnotetext{
21 Si hay o no un compromiso realista respecto de las leyes precisas, ése es un asunto que es irrelevante para mi argumento a favor de la autonomía del saber humanístico.
} 
sal bancaria estará abierta el sábado»- se retrasa, detiene, o simplemente se abandona -no se atribuye conocimiento-. En situaciones donde hay poco en juego (low stakes) se atribuye o autoatribuye conocimiento. En estas situaciones, si Ana se equivoca nada pasa respecto de su situación económica; en la otra clase de situaciones, Ana perdería mucho si piensa que sabe que el banco estará abierto pero resulta que no lo está, pues no habría depositado a tiempo los fondos que le permitirían cubrir sus deudas a primera hora del lunes.

Por supuesto que aquí tenemos una visión antiintelectualista del conocimiento y otros conceptos epistémicos, pues lo que parcialmente determina, en el sentido de hacer posible o permitir, la atribución de conocimiento o poder explicativo es la presencia y satisfacción de intereses prácticos.

Se puede criticar a este argumento en el sentido de que no se ve cómo puede tener éxito, pues cabe pensar que en situaciones donde hay mucho en juego, y poco a poco, al entrar en juego -y controlar la situación epistémica- factores exclusivamente epistémicos como evidencia, justificación y demás, se pueden poner bajo estricto control epistémico a los intereses prácticos. Más y mejor evidencia -llamar al banco antes que a un conocido para averiguar sus horarios sabatinos, una justificación más extensa-, varios testimonios y no sólo uno, harían posible una atribución y autoatribución de conocimiento positiva, antes que afirmar que intereses prácticos toman el control de la situación epistémica.

A pesar de esta salida intelectualista, en un caso más extremo que el recién expuesto, pero no por ello menos real y común, tendríamos a una automovilista que salva la vida si y sólo si se atribuye y atribuye conocimiento relevante a otros en el sentido siguiente. Ante lo que parece un accidente automovilístico, pero que en realidad es una ejecución 
de un asesino (sicario) pagado por un grupo poderoso del narcotráfico, nuestra automovilista se atribuye y atribuye conocimiento al asesino de que el accidente automovilístico no fue en realidad un accidente. Al hacer esta atribución puede actuar en consecuencia; el éxito o fracaso de su acción posterior, intento de huida, es otra cosa. No hay mucho tiempo para recolectar más y mejor evidencia o para armar una justificación más extensa; al atribuir esta pieza de conocimiento, y otras más, está en mejor situación para salvar su vida.

A diferencia del caso anterior, en una situación como ésta, donde el tiempo apremia una respuesta pronta, la determinación del conocimiento por parte de intereses prácticos parece mucho menos vulnerable a las dudas relacionadas con la disponibilidad ilimitada de tiempo y el cumplimiento de estándares epistémicos. La acción exitosa, a menos que defendamos la independencia de ésta de cualquier clase de conocimiento, nos indica que el conocimiento respectivo está presente.

Ya se sugirió una manera de entender la distinción entre intereses prácticos e intereses epistémicos: los primeros tienen que ver, aunque no exclusivamente, con ganancias y con pérdidas, mientras que los segundos se estructuran alrededor de la verdad y nuestras maneras de estudiar y entender los conocimientos que la constituyen.

Creo que se puede plantear el tema de los intereses epistémicos de varias maneras. A continuación caracterizo dos, pero tan sólo exploro y defiendo una de ellas con algunos detalles para mi argumento compatibilista. En primer lugar, a partir de la postulación de una teoría de los objetivos o metas del conocimiento ${ }^{22}$ se puede pasar a defender una concep-

$\overline{22}$ Ver Laudan, 1984; 1996; y discusión crítica en Cintora, 2005. 
ción de los intereses epistémicos adecuados que permitirían la obtención de aquéllos.

Otra posibilidad consiste en postular deberes epistémicos que se busca que tengan impacto directo en la motivación humana ${ }^{23}$. ¿Y cómo podría entregarnos una teoría de los deberes epistémicos una teoría de intereses de igual tipo?: a partir de una manera de entender el rol de un deber epistémico según el cual nuestros intereses epistémicos pueden pensarse como (conceptualmente) determinados por nuestros deberes epistémicos. El contenido del interés está determinado conceptualmente por el contenido del deber epistémico. Lo motivacionalmente factible, sin embargo, no es el postular deberes epistémicos que por sí mismos difícilmente nos lleven a pensar que poseen el poder de mover a los individuos a actuar de una manera $u$ otra, sino más bien intereses epistémicos que se pueden considerar como que efectivamente mueven a actuar a los agentes epistémicos a partir de estados psicológicos específicos en cada caso -deseos, inclinaciones, creencias, consideraciones, etcétera.

Una ventaja de una concepción tal de los intereses epistémicos es que dado que no es de ninguna manera incoherente pensar que los deberes epistémicos puedan entrar en conflicto entre sí, que el cumplimiento de un deber entre en conflicto con la realización de algún otro, una salida al respecto es que, en lugar de postular otros deberes que permitan la disolución de un conflicto entre deberes específicos, los intereses específicos de agentes epistémicos concretos pueden ser el recurso más adecuado para superar tales dificultades. Esto me parece bastante atractivo pues no son los deberes

23 Y si fuese el caso que existiesen metas epistémicas que determinan estos deberes epistémicos, entonces tendríamos a estos últimos como complemento de aquéllas. 
mismos los que entran en conflicto, éstos se cumplen o no, sino que es su promoción y realización en la conducta de los agentes epistémicos lo que indica conflicto y/o resolución del mismo.

En la reconstrucción de los deberes epistémicos que viene de Locke, y pasa por James y otros, se identifican al menos dos grandes deberes, a saber, el deber de creer lo verdadero -y por ende no creer lo falso-, por un lado, y el deber de formar, modificar y retener creencias verdaderas a la luz de evidencia, por el otro ${ }^{24}$. Digamos además que estas dos clases de deberes son bastante genéricas y se puede trazar su realización efectiva en deberes bastante concretos, según sea el caso de la investigación bajo consideración. Así, según la hipótesis que propongo, tendremos el interés en creer lo verdadero y el interés en formar, modificar y retener creencias a la luz de evidencia. Así tendríamos el interés en no creer contenidos falsos, lo cual sería un interés que nos movería, junto con el interés en modificar y retener creencias a la luz de la evidencia, en nuestra búsqueda de una explicación ulterior de muchas explicaciones heteronómicas. Este interés determina, pues, un interés en mejores explicaciones.

Además, a partir de nuestro interés en modificar nuestros contenidos epistémicos a la luz de la evidencia, con la finalidad de promover nuestro interés en contar con creencias verdaderas, también podría darse el caso de que algo que se aceptaba previamente como verdadero y definitivo sea visto como en necesidad de una explicación ulterior. Éste podría ser el caso con algunas afirmaciones de identidad entre fenómenos psicológicos o mentales y prácticas sociales

$\overline{24}$ Ver Richard Feldman, «The Ethics of Belief», para más detalles al respecto. 
(alimenticias) en donde al agregar información acerca de las bases neuroquímicas de tales fenómenos se comprende mucho mejor el funcionamiento de la mente/cerebro humano -como la que se sugiere en la descripción de la investigación de la anorexia nerviosa mediante estimulación magnética y de otros tipos del cerebro-. Así, nuestro interés en formar creencias a partir de evidencia más disciplinada genera explicaciones más demandantes bajo la forma de una generalización heteronómica que condensa una enorme cantidad de información, la cual, vamos a suponer, tiene un valor para ciertas disciplinas que buscan la explicación/verdad sobre la enfermedad, si bien todo ello puede resultar irrelevante para un agente.

Adviértase entonces que la caracterización de nuestros intereses epistémicos aquí propuesta es compatible tanto con nuestro saber heteronómico como con nuestro saber homonómico, una y otra clase de saber puede verse como motivada por estos intereses epistémicos y sería equivocado el afirmar que tan sólo una clase de saber, digamos el saber heteronómico, puede verse como motivado por aquéllos.

E incluso cabría defender la posibilidad de que nuestros intereses epistémicos sean más complejos y específicos de lo que nos indican nuestros dos intereses epistémicos genéricos. Por ejemplo, puede pensarse que nuestros intereses epistémicos están entremezclados con intereses prácticos -como los intereses en la autonomía o la integridad-, resultando en intereses mucho muy específicos que explicarían la actitud de quienes rechazan la posibilidad de enriquecer y complementar adicionalmente generalizaciones heteronómicas -y por ende explicaciones- aceptadas. Así, podría darse el caso de que se rechace la sugerencia antes mencionada como algo explicativo porque se piense que, no obstante que se tiene 
un interés en echar luz adicional sobre la psicología humana, eso es parcialmente incompatible con verse a sí mismo como un agente autónomo con dominio de sus emociones y estados fenoménicos, y un mero padecer un estado como la anorexia nerviosa parece incompatible con ello. Aunque un rechazo tal, habría que aclarar, podría obedecer a motivaciones absolutamente compatibles con nuestra hipótesis presente, es decir, que podrían existir intereses prácticos que nos mueven a buscar una explicación donde quede claro que mucho más tiene que decirse acerca del estado psicológico que la mera correlación con un estado neurofisiológico.

$\mathrm{Y}$ finalmente, pero no menos importante por ello, alguien podría objetar que los intereses epistémicos poseen, si acaso, el estatus de una inferencia a la mejor explicación y no un análisis riguroso que los defina de antemano. No veo mucha utilidad en tal cosa. Rechazo cualquier intención de llevar a cabo análisis conceptual de un concepto de interés epistémico; de hecho, dicha clase de análisis es un tipo de actividad filosófica que cada vez se ve con menos aprobación, y comparto muchas de las razones para verla así; sin embargo, acepto con reservas que se vea al concepto de intereses epistémicos aquí defendido como un caso de inferencia a la mejor explicación -y habría que argumentar esto más pormenorizadamente en otro lugar.

4. La visión compatibilista y la autonomía del saber humanístico

«Pero el saber heteronómico poco o nada tiene que ver con el saber humanístico en el argumento aquí defendido, y lo mismo parece suceder con el saber homonómico», alguien po- 
dría observar. No creo que esta observación fuera correcta, pues debería ser claro que en la medida en que la distinción homonómico/heteronómico, desde mi punto de vista, plantea una geografía no tradicional del saber a partir de los aspectos identificados en las generalizaciones típicas de ambas clases de saber -y si así se quiere, a partir de los conceptos expresados en esas generalizaciones-, y si el saber humanístico no es ajeno a características propias del saber heteronómico, entonces no puede afirmarse sin mayor argumentación que un saber nada tiene que ver con el otro -incluso concediendo, como tiene que ser, que el saber homonómico nada tiene que ver con las humanidades-. Lo importante, cabe insistir, es que la distinción homonómico/heteronómico hace posible pensar características centrales del saber y de las explicaciones que lo constituyen, de una manera que no resultan continentes de conocimiento sin contacto alguno. $Y$ la consecuencia inmediata es que si nada hay separado absolutamente, explicaciones absolutamente dispares y conceptos por completo ajenos los unos a los otros, entonces no hay razón para abrigar la existencia de algún «hueco epistémico» ${ }^{25}$ inevitable y permanente; a diferencia de los naturalismos moderno y sin adornos, y del espacio de las razones de McDowell, que sí operan bajo ese supuesto. Y si estoy en lo correcto al interpretar el pasaje de Davidson en la manera en que lo hago, entonces las propiedades de los distintos tipos de saber, así como los conceptos de entender, explicar y hacer

\footnotetext{
25 Obviamente al usar «hueco epistémico» quiero que venga a la mente del lector el hueco explicativo de que habla Levine (1983). Hay diferencias no obstante entre un hueco y otro; el epistémico tendría que ver con los dos grandes tipos de saber tal y como los supone McDowell, por un lado, y los fisicalistas reductivistas Loar, Kim y Lewis por el otro, mientras que el explicativo tan sólo tiene que ver con los estados de conciencia fenoménica y su explicación objetiva.
} 
más inteligible ${ }^{26}$, caen bajo la extensión de nuestros intereses epistémicos, y todo dependerá, en lo que respecta a quedar satisfecho con una explicación, de los intereses epistémicos subyacentes de los individuos que los aplican.

«De cualquier modo, parece que se acercan demasiado el saber humanístico y el científico-natural, tanto que parece que se borran las diferencias entre ellos», podría objetar alguien a mi argumentación a favor de una concepción compatibilista del saber. No, no es así; el saber científico-natural y el humanístico no se acercan, en ningún momento se identifica uno con el otro, o peor aún, se reduce; lo que siempre ha estado cerca y forma un continuo son las formas de comprender, explicar y prever. «En ese caso, parece que, de ser correcta la interpretación propuesta de la distinción homonómico/heteronómico al defender la continuidad entre el saber humanístico y el saber científico-natural, lo propio de la racionalidad, del espacio de las razones de McDowell, se va a la basura, pues las relaciones de justificación en el espacio lógico del que habla McDowell se reducen básicamente a distintas clases de explicación mediante algún tipo de razonamiento inductivo», nuevamente alguien podría objetar mi argumentación. Bueno, sí, pero es una cosa decir esto, y otra muy diferente decir que por el hecho de que haya formas de razonamiento inductivo en juego se aspire a alcanzar generalizaciones homonómicas o leyes acabadas. Lo cual no es algo que se quiera defender como inherente al saber hetero-

\footnotetext{
${ }_{26}$ El que sean conceptos relativos a los intereses de los agentes no es precisamente equivalente a decir que estos conceptos sean sensibles al contexto, como se repite en la actualidad. Incluso Kim afirma lo anterior: «[...] pero debemos tener en mente el hecho de que explicar es una noción maleable y frágil; algunos dirán que lo que cuenta como una explicación es altamente sensible al contexto además de un asunto pragmático (sino es que enteramente relativo o subjetivo) $[\ldots] »(\mathrm{Kim}, 2005 ; 130)$.
} 
nómico. Y esto indica toda una serie de diferencias entre distintas disciplinas, tanto al interior de las humanidades, como de las ciencias sociales, o incluso las científico-naturales, que se pueden considerar que vendrían determinadas por intereses epistémicos distintos.

« ¿Se buscaría eliminar la racionalidad propia del saber y la comprensión humanística con la distinción homonómico/heteronómico?», podría preguntar el defensor de las humanidades. No veo cómo; lo que sí se quiere es eliminar diferencias obsoletas entre la comprensión propia del saber humanístico y el saber científico-natural, como lo es aquélla que tiene que ver con el rechazo de la generalización heteronómica en las humanidades, o aquella otra que tiene que ver con la ausencia de vocabulario compartido. El objetivo, como se planteó al inicio, es mostrar que hay continuidad más allá de las diferencias inevitables en el vocabulario, en algunas de las relaciones de justificación, y en ciertos intereses. De ningún modo se busca subestimar estrategias de persuasión y convencimiento propias del debate argumentado como el experimento mental, las distintas clases de argumentaciones y los modos de interpretarlas, pero no es exclusivo de la filosofía todo ello; en la investigación científico-natural no son ajenos los experimentos mentales y los modos de interpretar los experimentos y las argumentaciones.

Tampoco, por otra parte, se busca reivindicar alguna suerte de homogeneidad metodológica, pues ésta parece ser una cosa, mientras que compatibilismo epistémico y metodológico es otra muy distinta; método homogéneo apunta a un sólo método, mientras que compatibilismo metodológico indica un número importante de métodos, tantos como disciplinas si así se quiere, que no son incompatibles entre sí. Y esta compatibilidad puede estimarse en términos de qué tan 
heteronómico es el saber en cuestión. Pero este punto también merece una discusión detenida aparte.

¿Cómo se puede ver que la distinción entre saber humanístico y saber científico-natural está contenida en aquella otra -lógicamente, por supuesto-?: en la medida en que la distinción homonómico/heteronómico davidsoniana nos permite construir un modelo del saber en su totalidad a partir de seis aspectos compartidos, y a partir de este modelo --que se puede formular lógicamente si se quiere- podemos obtener como consecuencias lógicas un número de casos de saber -principalmente saber humanístico, social y saber científiconatural-. Y debido a esta procedencia común hay continuidad y plena compatibilidad. Nunca superposición, como lo presuponen los fisicalistas reductivistas y sus enemigos.

Al ser de grado más que de naturaleza, tal y como sucede con aquella otra distinción, la autonomía del saber humanista puede plantearse, por lo tanto, de manera menos dramática de lo que usualmente se hace, y sin las consecuencias, nefastas desde mi punto de vista, de intentar aislar el saber humanístico del saber científico-natural. La autonomía de las humanidades tiene más que ver con los intereses que mueven sus investigaciones, con los intereses que nos llevan a preferir un vocabulario y unas explicaciones que con una metodología o visión supuestamente única y exclusiva.

\section{BIBLIOGRAFÍA}

Cintora Gómez, A. (2005). Los presupuestos irracionales de la racionalidad. Barcelona: Rubí \& Anthropos \& Universidad Autónoma Metropolitana-Iztapalapa. 
Davidson, D. (1970a). «Events as Particulars». En: Essays on Actions E Events, Oxford: Clarendon Press. (Hay traducción al español en UNAM/Crítica, 1995.) . (1970b). «Mental Events». En: Essays on Actions $\mathcal{E}$ Events.

Feldman, R. (2000). «The Ethics of Belief». Philosophy and Phenomenological Research 60; 667-695.

Herstein, G.L. (2005). «Davidson On the Impossibility of Psychophysical Laws». Synthese 145; 45-63.

Kim, J. (2003). "Philosophy of Mind and Psychology». En: Ludwig, K. (ed.), Donald Davidson. Cambridge: Cambridge University Press.

. (2005). Physicalism, Or Something Near Enough Kim.

Princeton, N.J.: Princeton University Press.

King's College, London Institute of Psychiatry \& NHS 2010, Study Reference 10/H0807/16: Transcranial Magnetic Stimulation for Anorexia Nervosa.

Laudan, L. (1984). Science and Values. Berkeley, Cal.: University of California Press.

. (1996). Beyond Positivism and Relativism. Boulder,

Col.: Westview Press.

Levine, J. (1983). «Materialism and Qualia: The Explanatory Gap». Pacific Philosophical Quarterly 64; 354-361.

Lewis, D. K. (1994). «David Lewis: Reduction of Mind». En: Guttenplan, S. (ed.), A Companion To the Philosophy of Mind. Oxford: Blackwell.

Loar, B. (1981). Mind and Meaning. Cambridge: Cambridge University Press.

McDowell, J. H. (1994). Mind E World. Cambridge, Mass.: Harvard University Press. 
. (2004). «Naturalism in the Philosophy of Mind». En: De Caro, M. \& D. Macarthur (eds.), Naturalism in Question, Cambridge, Mass.: Harvard University Press.

Ramberg, B. (1999). «The Significance of Charity». En: Hahn, E. L. (ed.), The Philosophy of Donald Davidson, Chicago \& LaSalle, Illinois: Open Court.

Simons, P. (2003). «Events». En: Loux, M. J. \& D. W. Zimmerman (eds.), The Oxford Handbook of Metaphysics, Oxford \& New York: Oxford University Press.

Stanley, J. (2005). Knowledge and Practical Interests. Oxford: Oxford University Press.

Wright, G. H. (1971). Explicación y comprensión. Tr. Luis Vega R. Madrid: Alianza Universidad. 


\section{RESUMEN}

En el presente ensayo se defiende que, además de la diferencia obvia entre sus vocabularios respectivos, hay muchas menos diferencias entre el saber científico-natural y el saber humanístico de las que usualmente se reconocen. Esto no quiere decir, ni mucho menos es equivalente a afirmar, que hay reducción teórica, metodológica u ontológica, o que no tenga sentido hablar de la autonomía conceptual del saber humanístico. De este modo, hay continuidad entre la filosofía, el resto de las humanidades, y la investigación científiconatural; y esto no es incompatible con la autonomía del saber humanístico. Una interpretación de la distinción entre saber homonómico y saber heteronómico proporciona una plataforma para explorar una visión compatibilista entre el saber humanístico y el saber científico-natural. Las diferencias no lingüísticas entre saberes son más de grado que de naturaleza. La autonomía del saber humanista, consecuentemente, puede replantearse sin tentaciones revisionistas.

Palabras clave: ciencias naturales; humanidades; Davidson; saber homonómico; saber heteronómico.

\section{ABSTRACT}

In this paper I argue that, besides the obvious distinctions in their respective vocabularies, the differences between humanistic and scientific knowledge are thinner than is usually recognized. This doesn't mean, and is not equivalent to the claim, that there is a theoretical, methodological or ontological reduction, or that is senseless to talk about the conceptual autonomy of humanistic knowledge. In this sense, there is 
continuity between philosophy, the rest of the humanities, and scientific inquiry; this is not incompatible with the autonomy of humanistic knowledge. An interpretation of the distinction between homonomic and heteronomic knowledge sets the stage for a compatibilist view of humanistic and scientific knowledge. Non-linguistic differences among disciplines are of grade rather than of nature. Humanistic autonomy, in consequence, can be restated without revisionist temptations.

Key words: natural sciences; humanities; Davidson; homonomic knowledge; heteronomic knowledge. 
